九州大学学術情報リポジトリ

Kyushu University Institutional Repository

\title{
Development of a Broadband Telemedical Network Based on Internet Protocol in the Asia-Pacific Region
}

Nakashima, Naoki

Department of Medical Informatics, Kyushu University Faculty of Medicine

Shimizu, Shuji

Department of Endoscopic Diagnostics and Therapeutics, Kyushu University Faculty of Medicine

Okamura, Koj i

Computing and Communications Center, Kyushu University

Hahm, J. S.

Department of Medicine, Hanyang University Medical College

他

http://hdl. hand le. net/2324/12823

出版情報: Methods of Information in Medicine. 46 (6), pp.709-715, 2007-12. Schattauer バージョン：

権利関係 : (C) 2007 Schat tauer GmbH. 


\title{
Development of a Broadband Telemedical Network Based on Internet Protocol in the Asia-Pacific Region
}

\author{
N. Nakashima ${ }^{1}$, S. Shimizu' ${ }^{2}$ K. Okamura ${ }^{3}$, J. S. Hahm ${ }^{4}$, Y. W. Kim ${ }^{5}$, H. S. Han ${ }^{6}$, N. Torata ${ }^{7}$, \\ Y. Antoku', Y. S. Lee ${ }^{8}$, M. Tanaka \\ 'Department of Medical Informatics, Kyushu University Faculty of Medicine, Fukuoka, Japan \\ ${ }^{2}$ Department of Endoscopic Diagnostics and Therapeutics, Kyushu University Faculty of Medicine, \\ Fukuoka, Japan \\ ${ }^{3}$ Computing and Communications Center, Kyushu University, Fukuoka, Japan \\ ${ }^{4}$ Department of Medicine, Hanyang University Medical College, Seoul, Korea \\ ${ }^{5}$ Research Institute and Hospital, National Cancer Center, Goyang, Gyeonggi, Korea \\ ${ }^{6}$ Department of Surgery, Seoul National University Bundang Hospital, Seoul, Korea \\ ${ }^{7}$ Department of Surgery and Oncology, Graduate School of Medical Sciences, Kyushu University, \\ Fukuoka, Japan \\ ${ }^{8}$ Medical Media and Information Technologies, Stanford University, Palo Alto, California, USA
}

\section{Summary}

Objectives: To promote the exchange of knowledge and standardization of medical procedures and medical systems in the Asia-Pacific region, we established a medical network with high-quality moving images over broadband Internet lines in February 2003.

Methods: Real-time teleconferences and live demonstrations with medical-quality videos, broadcast via the Digital Video Transport System, have been used to teach surgical techniques and other medical procedures across national borders.

The Asia-Pacific Advanced Network (APAN) committee in August 2005 formally approved our proposal to establish a medical working group within APAN. The network was expanded by the launch of the TransEurasia Information Network 2 in 2006 . By the end of 2006, we had conducted 82 events, in 10 countries in the Asia-Pacific region. The multi-station event has increased every year.

Results: There have been no serious transmission problems or ethical conflicts so far. With these experiences and current achievements, we hope to extend this advanced network system to the entire Asia-Pacific. Conclusion: This system is a promising and very useful tool for the standardization of medical system and procedures across national borders. Drawing upon these experiences and current achievements, we hope to extend this advanced network system to the entire AsiaPacific region.

\section{Keywords}

Telemedicine, international educational exchange, medical informatics

Methods Inf Med 2007; 46: 709-715

\section{Introduction}

Medical care varies between countries because of disparities in economic power and differences in policies, religions, and customs. Medical information is not easily disseminated beyond national borders, and medical staff are rarely aware of the existence of these differences. This problem can be solved in part by establishment of a teleconference system that can transmit highquality moving images [1].

This is a new network communication system that can transmit real-time, highquality moving digital video (DV) images over Internet Protocol (IP). We are now able to use international submarine fiber-optic cable networks in the Asia-Pacific region for broadband transmission. The distribution of medical information with DV over IP beyond national borders makes medical staff keenly aware of the differences in medical services and uncovers the relative advantages and disadvantages in the medical field between countries. For example, via the network system, medical staff can gain concrete and exact knowledge of advanced medical techniques and devices developed or used in other countries without a large cost or loss of time. In the case of outbreaks of emerging infectious diseases such as bird flu and severe acute respiratory syndrome (SARS), and in the eventuality of ter- rorism with biochemical weapons in the Asia-Pacific region, we may grasp the reality of strange symptoms in remote areas and prevent the spread of the disease and prepare for the care of sufferers.

Herein, we describe how we have expanded the Asia-Pacific international medical network since February 2003 and describe the issues we have had during the expansion of the network.

\section{Methods}

\subsection{Organization}

The Hyeonhae/Genkai project was established in 2002 for development of informatics research and friendship between Korea and Japan via the Korea-Japan Cable Network (KJCN). In February 2003, as a subproject of the Hyunhae/Genkai project, Japanese and Korean medical groups collaborated to use the KJCN for medical purposes $[2,3]$.

\subsection{Networks}

\subsubsection{Domestic Network in Japan}

We connected conference rooms and operating rooms at Kyushu University Hospital, 


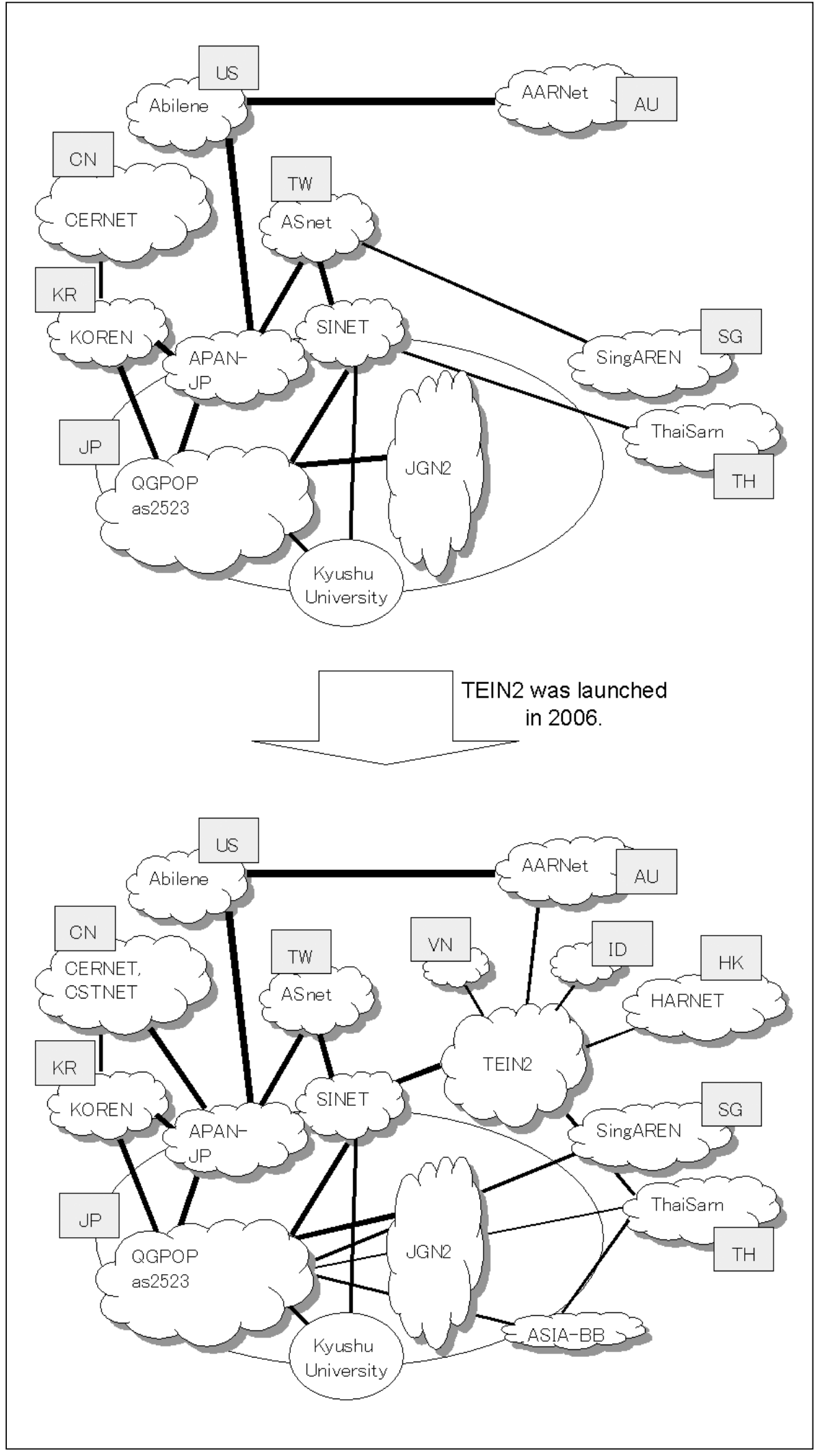

Fig. 1 The Asia-Pacific international network configuration before and after TEIN2 was launched in 2006. Connected countries are shown by abbreviations (JP: Japan, KR: Korea, US: United States, AU: Australia, CN: China, TW: Taiwan, TH: Thailand, SG: Singapore, VN: Vietnam, ID: Indonesia, and HK: Hong Kong).
Japan, to the Computing and Communications Center (CCC) of the main campus of Kyushu University via the campus network at $1 \mathrm{Gbps}$, which we increased to $10 \mathrm{Gbps}$ in March 2006. Kyushu Giga POP (QGPOP), which the CCC manages, functions as the primary hub of the network in this project. We connected from QGPOP to domestic giga-class backbones, the Japan Gigabit Network (JGN) (until 2003), JGN2 (since 2004) [4], and the Super Science Information Network (Super SINET [5]). We connected Iwate Medical College (Morioka), Nagasaki University Hospital (Nagasaki), Fujimoto Hayasuzu Hospital (Miyakonojo), and academic meeting venues in Sapporo, Tokyo, Yokohama, and Fukuoka, Japan.

\subsubsection{International Optic Cables and Domestic Networks in Other Countries}

We used public optic submarine cables for international connections and for domestic research and educational networks within other countries (Fig. 1). We connected the QGPOP network and the Korea Advanced Research Network (KOREN [6]) in Korea, via the Asia-Pacific International Infrastructure (APII) (1 Gbps), which uses the KJCN. In China, we connected to the China Education and Research Network (CERNET [7]) through the KOREN-CERNET submarine connection (155 Mbps). In Taiwan, we used the Academic Service Network (ASnet [8]), with the connection from Super SINET or Asia-Pacific Advanced Network-Japan (APAN-JP) at 1 Gbps through an optic submarine cable, which was prepared by ASnet. In Thailand, we connected to the Thai Social/Scientific, Academic and Research Network (ThaiSarn [9]) via Super SINET (45 Mbps). Australia's Research and Education Network (AARNet [10]) was connected to Abilene [11] through transPAC [12] from APAN-JP. We did not have a direct connection with Singapore; thus, we used a combination of the JapanTaiwan connection and the ASnet-Singapore connection (155 Mbps), using the Advanced Research and Education Network (SingAREN [13]) in Singapore.

Trans-Eurasia Information Network 2 (TEIN2), which was launched in 2006, made connection to South Asian countries 
easier. We connected Vietnam, Hong Kong, and Indonesia in the summer of 2006. We also changed the route to Singapore and Thailand in 2006, as we were able to directly connect to these countries via JGN2. Additionally, we used the Asia Broadband program line (ASIA-BB [14]) for the connection to Thailand starting in late 2005.

\subsection{Terminal Systems Organiza- tion for Moving Image Transmission}

For transmission of moving images with standard definition, we have used DV over IP in this network. We usually use the Digital Video Transport System (DVTS) over IP. DVTS is open source freeware, downloadable from a Web site [15]. We also transmit High Definition Television (HDTV) images compressed by Mpeg2 on the network. We sometimes use Virtual Network Computing to transmit high-definition static images (e.g., images for pathologic diagnosis). We always have ready ITU H.323 Polycom series (Polycom Inc.) or PCS-1 (Sony Corp.) as a backup system.

When an event has multiple stations, we use a DV4 system (Qubit Systems Inc.) that can integrate four DV signals into one digital image without analog conversion. In the APAN-Tokyo meeting in January 2006, for which we connected ten stations between six countries, we had a chance to use a DV10 system that can integrate ten DV signals. For effective teleconferencing, we always transmit moving images and voice in both directions, and we often use additional transmission to show other images.

\subsection{Security System}

When we transmit live images of patients, we obtain written informed consent from them. In these live transmissions, we used C4S-VPN (Focus Systems Co. through 2003), and AR550S (Allied Telesis Co.) after 2003 as security systems that were approved for use by the ethics committee of Kyushu University Graduate School of Medicine.

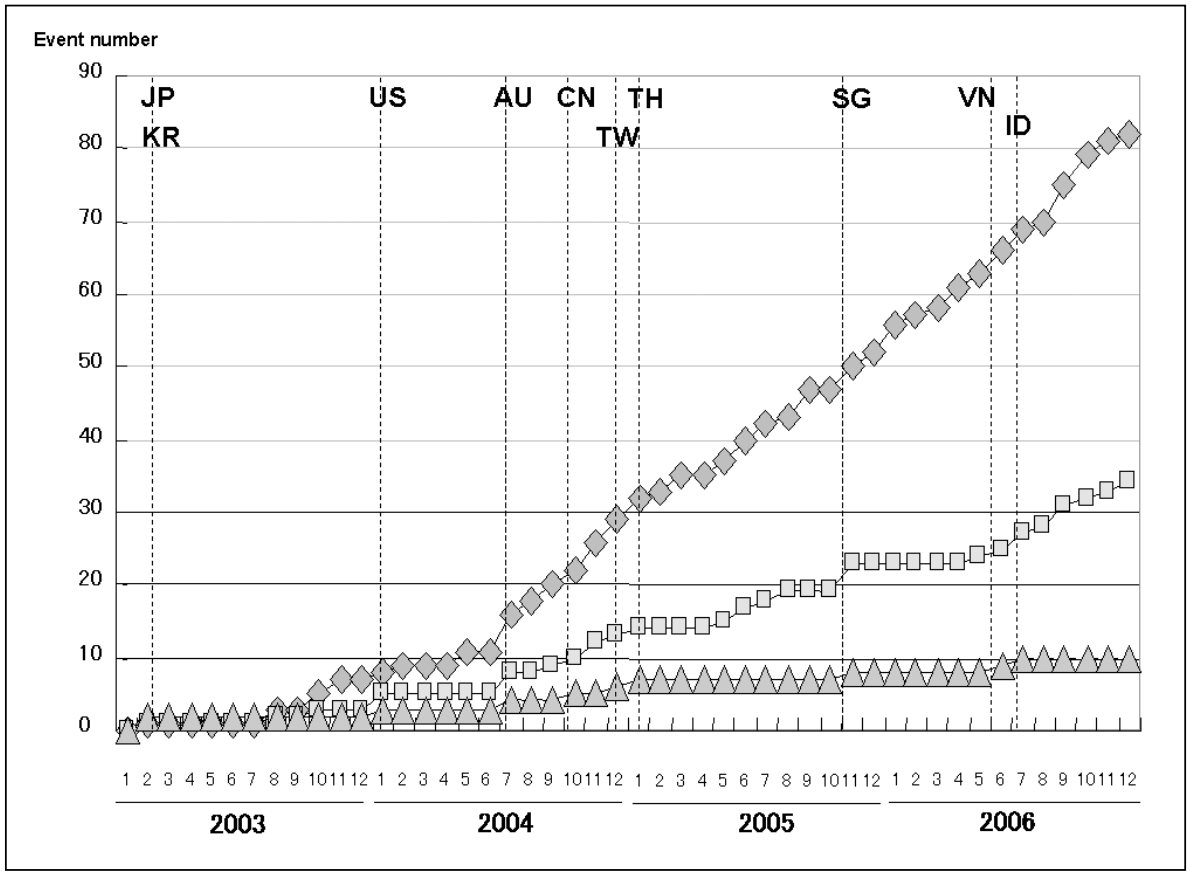

Fig. 2 Record of project events (2003-2006). Connected countries are shown by abbreviations (JP: Japan, KR: Korea, US: United States, AU: Australia, CN: China, TW: Taiwan, TH: Thailand, SG: Singapore, VN: Vietnam, and ID: Indonesia). Diamonds represent events, squares represent connected institutions, and triangles represent connected countries.

\section{Results}

\subsection{Evaluation of the System}

Installing the system and connecting to the network are inexpensive if a broadband line is available at the venue. In the minimum setup, we do not need any special equipment except two PCs and one DV camcorder. Depending on the size, importance, and technical difficulty of the event (e.g., multistation event and/or live demonstration), we need other equipment, such as an echo canceller, audio mixer, DV mixer, and VPN router. We sometimes need to contract additional specialists in network engineering and audio control, including their equipment for special events such as international academic events, which can cost US $\$ 10,000$ - $\$ 20,000$ per event.

The quality of transmitted moving DVTS images was as good as that of the original digital video. A frame rate of 30 per second was obtained by $30 \mathrm{Mbps}$ bandwidth, and the images were smooth and not sluggish. The sound was clear; however, there audio jitter was present during the entire course due to packet loss. The time delay was less than $0.3 \mathrm{sec}$ between Japan and Korea, and slightly longer for other countries (maximum $1.0 \mathrm{sec}$ ), which made for minimal to no stress at each endpoint.

\subsection{System Usage Record}

The event record is shown in Figure 2. The first event was conducted between Hanyang University (Korea) and Kyushu University Hospital (Japan) in February 2003. In 2003, we established the basic scope of the project (organization, network configuration, and application). For example, we decided to use security software to protect patient privacy during the first live transmission of a patient's image in August 2003.

We joined the APAN consortium in January 2004 (APAN-Honolulu). After three subsequent demonstrations of telemedical conferencing during APAN meetings, which are held twice a year, the medical working group was formally approved by APAN by a vote during the APAN-Taipei 


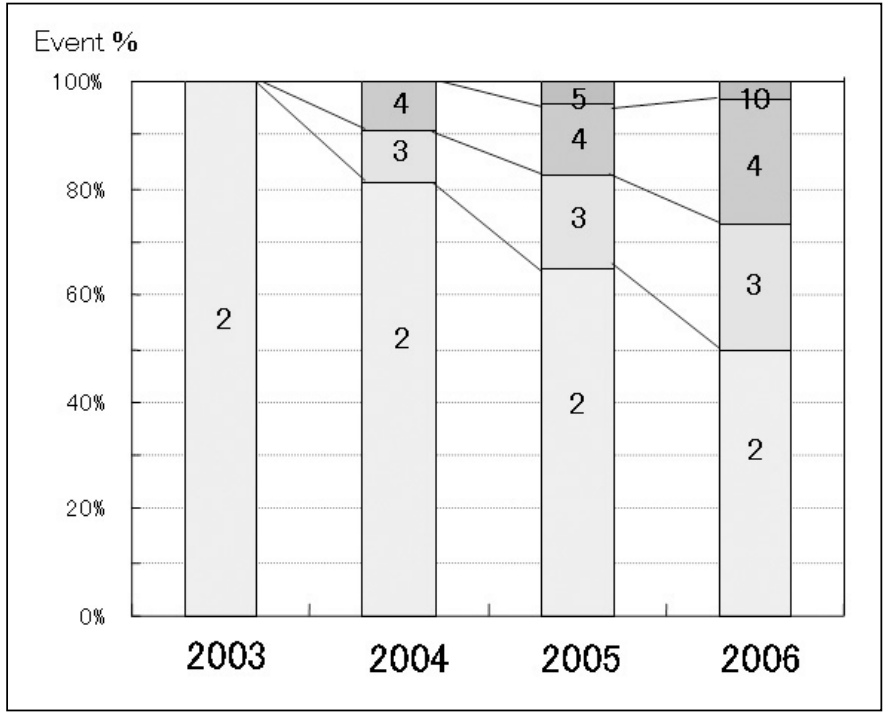

Fig. 3

Percentages of multi-station events per year. The numbers in the bars represent the number of stations per event.

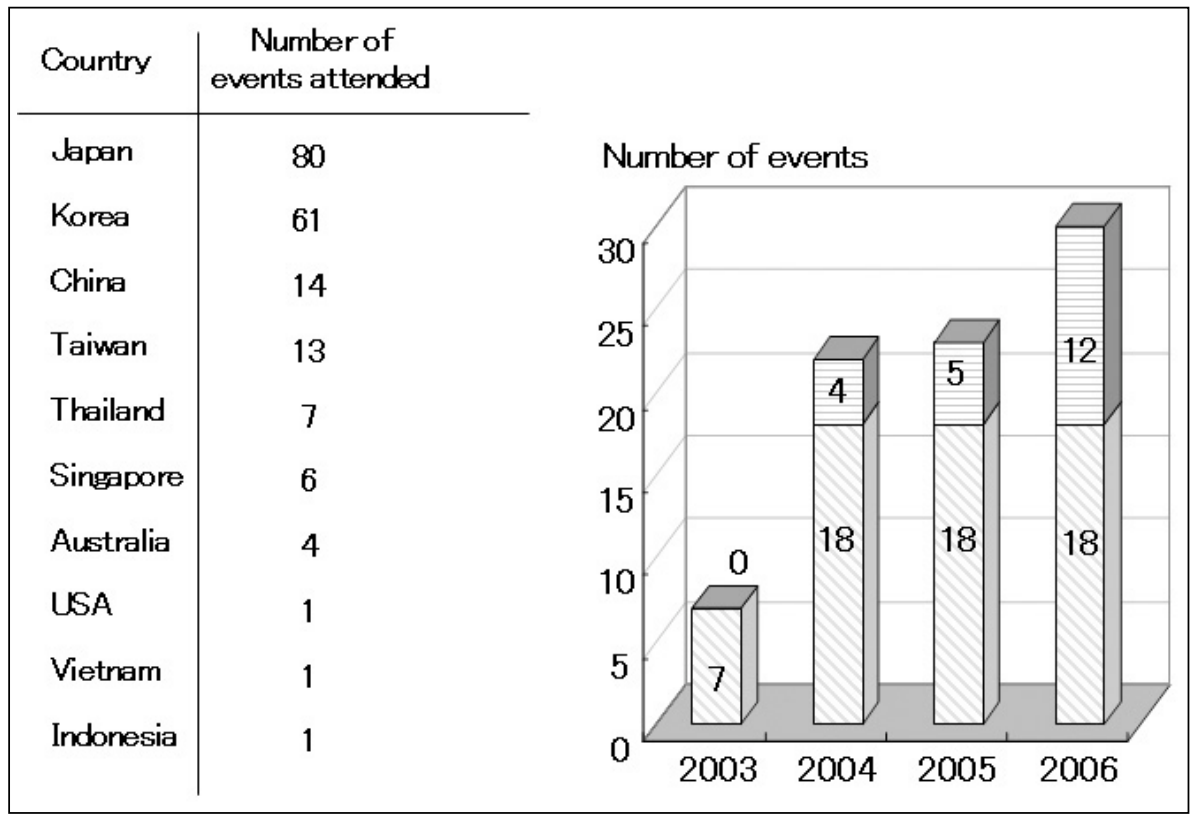

Fig. 4 Left: number of events attended by each country (2003-2006). Right: number of events Korea attended (column of diagonal lines) and did not attend (column of parallel lines) per year. Japan participated in all events except two in 2006.

meeting in August 2005 [16]. We met many medical doctors/researchers at each APAN meeting and therefore built a human network to help expand our project. We also gained partner institutions in Asia-Pacific countries through our attendance at each APAN meeting by asking different institutions to be host stations. Thus, we established multiple stations during and after each APAN meeting by adding new inducted a constant number of events over the past four years, for a total of 82 by the end of 2006 (Fig. 2). The percentage of multistation (three stations or more) events increased each year, reaching 50\% in 2006 (Fig. 3), although the level of difficulty of these events is high.

Japan and Korea have been the main participants in the project (Fig. 4, left), fol- lowed by China and Taiwan. Participation by South Asian countries has clearly increased since the launch of TEIN2 in 2006. Japan and Korea have maintained steady participation (Fig. 4, right).

Of the past 82 events, 26 were live transmissions of patient images and 37 were transmissions to academic meeting venues, 13 of which overlapped, that is, 13 involved live transmission of patient images to academic meeting venues (Fig. 5). The other 32 events were regular teleconferences between conference rooms.

\subsection{Categories of Event}

\subsubsection{Transmission of Live Images of Patients to a Conference Room or Office}

For demonstration of medical procedures, we have transmitted i) live endoscopic surgeries, ii) live microscopic surgeries, and iii) live endoscopic examinations from the operating room or examination room. The surgeon can hear and talk to other stations during the procedure. We usually create a separate station in the surgeon's institution for explanation of the procedure to avoid disturbing the surgery. Usually, the surgeon usually has a high level of experience with the technique or is using a cutting-edge device. Another use of transmission of live images is telementoring. For example, we connected an operating room at a hospital in Seoul with a conference room at Kyushu University Hospital in Japan in 2004. In Seoul, doctors were performing microscopic surgery for an orbital tumor, a rare disease. A neurosurgeon at Kyushu University Hospital who is a specialist in this type of tumor was able to advise the surgical team during the surgery on the basis of clear moving images transmitted from Korea to Japan; a successful operation resulted.

\subsubsection{Transmission to Academic Meeting Venues}

It is difficult for medical staff to attend academic meetings abroad because they cannot leave their patients for extended periods of time. With our system, we have conducted many remote lectures or presentations, 
making it possible for medical staff to remotely attend a meeting from their own hospital. We also used this system for virtual tours of remote institutions. In November 2005 , we held a teleconference at the 25th Joint Conference of Medical Informatics in Yokohama, Japan. In this session, we provided a virtual tour of Seoul National University Bundang Hospital, which is famous worldwide as an e-hospital. More than 260 attendees had the opportunity to see an advanced electronic medical record system firsthand (Fig. 6).

\subsubsection{Live Tramsmission of Patient Images to Academic Meeting Venues}

We have transmitted live demonstrations of medical procedures in actual patients to international academic meeting venues. We are able to introduce a procedure to hundreds of specialists in the field in different countries. For example, we connected the venue of the 94th Annual Meeting of the Japanese Urological Association in Fukuoka, Japan, and an operating room at Hanyang University Hospital in Seoul, Korea, and transmitted a live endoscopic urological surgery to the venue, where more than 1000 urologists were present. They were able to learn about the procedure and discuss the surgery with doctors at the Korean station.

\subsubsection{Regular Teleconferencing}

"Regular teleconferencing" refers to the transmission of a closed-type conference without live images of patients. For regular teleconferences, the participants discuss medical systems and procedures (techniques, equipment, etc.) for one hour to five hours. For example, a cholangio-pancreatic clinicopathological (CPC) conference has been held monthly at Kyushu University Hospital for more than ten years. In February 2005, we started a regular CPC teleconference between Seoul National University Bundang Hospital and Kyushu University Hospital, and we have added about three regular teleconferences per year. In the CPC teleconferences, anonymous patient histories and preoperative images were shown along with still images. The quality of the images was as good as that of the original

Fig. 5

Classification of broadband medical teleconferences.

The numbers represent events included in our project (2003-2006).
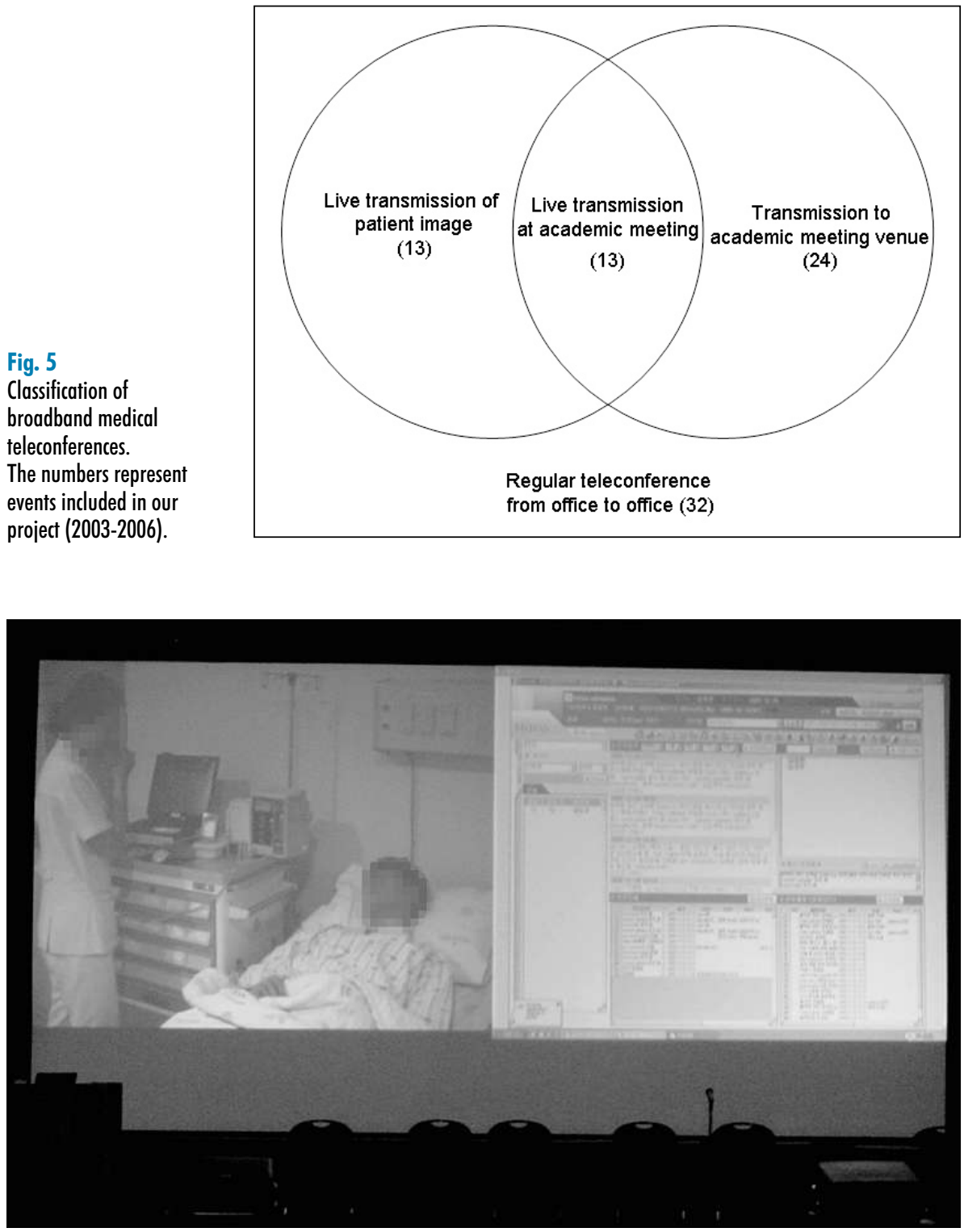

Fig. 6 The screen shows a virtual visit to Seoul National University Bundang Hospital and an electronic medical record system, which were provided at the 25th Joint Conference of Medical Informatics in Yokohama, Japan. Left: nurse caring for a patient (actor). Right: human interface of an electronic medical record system

digital video. The surgical anatomy was correctly identified, and the procedures were well understood. We conducted 32 regular medical teleconferences by the end of 2006 .

\section{Discussion}

The ITU H series epitomized by H.323 is a highly mature, distributed network telecon- ferencing system, which includes a mechanism for call alerting (analogous to a telephone ring) and a means of using various bandwidths. However, the moving images can be of insufficient quality because H.323 is designed for low- to moderate-speed bandwidth connections ( $<2 \mathrm{Mbps})$. We do not think the quality of moving images is adequate for medical purposes. Because our activities are aimed at developing a nextgeneration networking technology, we are attempting to transport high-quality moving 
images as DV images for use in the medical field.

Since the early 1990s, medical videoteleconferences with high-quality video have been conducted worldwide by private network with asynchronous transfer mode technology [17]. However, it costs too much to construct a network for each event (usually more than US $\$ 100,000$ ). Compressed DV images, mainly in Mpeg format, were transmitted over IP starting in the late 1990s to reduce costs [18, 19]. However, the compression process adds time, and this delay affects the communication negatively. To avoid this negative effect, we started to transmit uncompressed images.

The Internet Engineering Task Force (IETF) has standardized the data format to transport DV by RFC3189 over a highspeed network. This protocol has been incorporated into software (e.g., DVTS) and hardware (e.g., DVCommXP). Our experience strongly suggests that DVTS is a candidate for use in a next-generation teleconferencing system. In fact, Access Grid, a wellestablished multi-station teleconferencing system, uses DVTS as a transport system.

The present system, which processes high-quality moving images, will help remove barriers to communication between countries in the Asia-Pacific region and will help standardize medical techniques, devices, medicines, policies, and ethics.

This network project in Asia-Pacific region has played two roles with respect to satisfactory growth of the network. First, it has served as the hub of the telemedical network within the Asia-Pacific region. The project promoted the exchange of medical knowledge only between Japan and Korea in the first year. However, since our involvement in several APAN meetings, many more Asia-Pacific countries have participated in the project. We have already connected ten countries, and we plan to connect the Philippines, India, Malaysia, and New Zealand in 2007.

Second, it has functioned as the communication center for medical knowledge and network technology. Those in the medical field want to use advanced information and communication technologies for multimedia and high-speed transmission of highquality moving images to exchange medical knowledge with their colleagues in remote places. However, such contact has been sporadic or not well integrated. The online and offline activities of this project function to close the gap between well-served and underserved areas.

We are extending the medical network with DVTS over IP in the Asia-Pacific region. The numbers of events and connected institutions have increased rapidly over the past four years. We found that DVTS has several advantages for use in broadband medical networks.

1) DVTS is open source freeware, downloadable from a Web site [15]. Set-up is minimal; only two PCs with a DV port and a commercially available DV camera are needed for each terminal.

2) For medical purposes, images should be of high quality. DVTS can transmit highquality moving images (standard definition level).

3) Because there is no compression process in DVTS, it produces only a short delay, less than $0.3 \mathrm{sec}$ in the case of transmission between Japan and Korea. The delay is short enough to facilitate natural discussion, not compounding the difficulties already present due to language barriers.

4) With DVTS, multiple channels are easily created with DV4 and DV10. In telemedicine, it is important to gather information from multiple sources.

DVTS has the following limitations:

1) DVTS needs a 30-Mbps transmission bandwidth to send a full-frame image for a single channel. Further expansion of the network is limited by the available bandwidth in a particular area.

2) DVTS has a complicated human interface to be used by the medical staff. Thus, training and technical support are necessary.

3) DVTS supports transport of high-quality moving images; however, the transmitted sound is not of equal quality. Packet loss causes audio jitter. We have often used alternative transmission for sound by PC-1 or Skype [20].

4) DV4 and DV10 are compatible with NTSC (National Television Standards Committee), but not with PAL (Phase
Alternating Line), which is the standard protocol for television transmission in Australia, China, and Thailand. Thus, we send Japanese equipment to partner stations for use with DV4 or DV10 in these countries.

In the use of DVTS with or without security software, there were no major communication or ethical problems up to the end of 2006.

In the future study, we will add other technology to our system to improve telecommunication efficiency. For example, we want to try transmission of high-definition moving image, stereoscopy image [21], and image with computer processing technology [22]. We also want to spread system users to patients and citizens in Asia-Pacific region for their health management. Recently, matured video teleconference system was proved to support sophisticated education as a tele-health management system [23].

In conjunction with participation in APAN meetings, we have established a high-quality video transmission system over IP throughout the Asia-Pacific region that is easy to use, reliable, and economical. Currently, the minimal requirement for DV transmission for telemedicine is $30 \mathrm{Mbps}$ per channel. This system is a promising and very useful tool for the standardization of medical systems and medical procedures in the Asia-Pacific region.

\section{Acknowledgments}

We express our sincere thanks to Network NOC members of APII, KOREN, and QGPOP. We also appreciate the engineering staff of Kyuden Infocom Company for continuous support of the project.

This project has been supported in part by the Ministry of Internal Affairs and Communications as an "e! project", and by the "Development and Operation of the Next Generation Internet Technologies", one of the Core University Programs funded by the Japan Society for the Promotion of Science.

\section{References}

1. Takahashi T. The present and future of telemedicine in Japan. Int $\mathrm{J}$ Med Inform 2001; 61: 131-137.

2. Shimizu S, Hahm JS, Kim YW, Park YJ, Lee JH, Youm SK, Okamura K, Nakashima N, Choi HS, Kang CH, Kawamoto M, Tanaka M. Telemedicine with digital video transport system over the 
The Asia-Pacific Broadband Telemedical Network

Korea-Japan cable network. Korean Med Inform 2003; 9: S322-326.

3. Nakashima N, Okamura K, Hahm JS, Kim YW, Mizushima H, Tatsumi H, Moon BI, Han HS, Park YJ, Lee JH, Youm SK, Kang CH, Shimizu S. Telemedicine with digital video transport system in Asia-Pacific area. Proceedings of Advanced Information Networking and Applications (AINA) 2005; 1: 253-257.

4. JGN; http://www.jgn.nict.go.jp/index.html

5. super SINET; http://www.sinet.ad.jp/s_sinet/ index.html

6. KOREN; http://www.koren21.net/eng/index.php

7. CERNET; http://www.edu.cn/HomePage/ english/index.shtml

8. ASnet; http://www.asnet.net.tw

9. ThaiSarn; http://thaisarn.nectec.or.th/htmlweb/ index.php

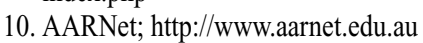

11. Abilene; http://abilene.internet2.edu

12. transPAC; http://www.transpac.org

13. SingAREN; http://www.singaren.net.sg/start.php

14. ASIA-BB; http://www.dosite.jp/asia-bb/en/ index.html
15. DVTS; http://www.dvts.jp

16. Nakashima N, Shimizu S, Okamura K, Hahm JS, Kim YW, Moon BI, Han HS, Lee JH. Rapid growth of broad-banded telemedicine network in Asia-Pacific area. Proceedings of the 7th ChinaJapan-Korea Medical Informatics Conference 2005. pp 35-37.

17. Elion JL, Petrocelli RR. A high-speed network for cardiac image review. Proc Annu Symp Comput Appl Med Care 1994. pp 428-432.

18. Boudier T, Shotton DM. Video on the Internet: An introduction to the digital encoding, compression, and transmission of moving image data. J Struct Biol 1999; 125: 133-155.

19. DeAngelis GA, Dempsey B, Berr S, Fajardo LL, Sublett J, Hillman BJ, Weaver A, Berbaum K, Dwyer SJ. Diagnostic efficacy of compressed digitized real-time sonography of uterine fibroids. Acad Radiol 1997; 4: 83-89.

20. Skype; http://www.skype.com

21. Szold A. Seeing is believing: visualization systems in endoscopic surgery (video, HDTV, stereoscopy, and beyond). Surg Endosc 2005; 19: 730-733.
22. Kruger S, Vogt F, Hohenberger W, Paulus D, Niemann H, Schick CH. Evaluation of computerassisted image enhancement in minimal invasive endoscopic surgery. Methods Inf Med 2004; 43: 362-366.

22. Moehr JR, Anglin CR, Schaafsma JP, Pantazi SV, Anglin S, Grimm NA. Video conferencing-based telehealth - its implications for health promotion and health care. Methods Inf Med 2005; 44: 334-341.

\section{Correspondence to:}

N. Nakashima, MD, PhD

Department of Medical Informatics

Kyushu University Faculty of Medicine

Maidashi 3-1-1, Higashi-ku

Fukuoka 812-8582

Japan

E-mail: nnaoki@info.med.kyushu-u.ac.jp 